\title{
Immobility due to COVID-19 confinement, use of contraceptives and presence of the G20210A mutation. Validity of the Virchow triad for the presentation of Deep Venous Thrombosis and Pulmonary Thromboembolism.
}

Luz Casique-Bocanegra ( $\sim$ locasique@saludcastillayleon.es )

Hospital Universitario Virgen de la Concha

Aranzazu Rodríguez-Garrote

Hospital Universitario Virgen de la Concha

Gaisha Danabayeva

Hospital Universitario Virgen de la Concha

Javier Alonso-Díaz

Hospital Universitario Virgen de la Concha

Noelia Diez-Martín

Hospital Universitario Virgen de la Concha

\section{Laura Gonzalez-Tejedor}

Hospital Universitario Virgen de la Concha

Eva Lumbrera-Moreno

Hospital Universitario Virgen de la Concha

Marcos Edgar Fernández-Cuadros ( $\square$ marcosefc@hotmail.com )

Hospital Universitario Santa Cristina https://orcid.org/0000-0001-6153-9075

\section{Research Article}

Keywords: Virchow's triad, thrombosis, pulmonary embolism, deep vein thrombosis, thrombophilia, prothrombin, G20210A mutation, hereditary thrombophilia, immobility, confinement, COVID-19

Posted Date: December 20th, 2021

DOI: https://doi.org/10.21203/rs.3.rs-1104706/v2

License: (c) (i) This work is licensed under a Creative Commons Attribution 4.0 International License.

Read Full License 


\section{Abstract}

Venous thromboembolic disease is a complex and multifactorial pathology, the result of the interaction of both genetic and environmental factors. The Virchow triad, first described in 1859, is still valid to explain the pathogenesis of thrombosis, where three main factors are reflected: a) hypercoagulable state; b) impaired blood flow; c) endothelial injury. The outbreak of a new COVID-19 pandemic has led to drastic confinement measures, with the consequent syndrome of immobility, as occurred in Spain between the months of March to June 2020. Pregnancy and hormonal contraception have proven to be an environmental factor predisposing to venous thrombosis. The presence of genetic factors, such as the mutation of the prothrombin gene G20210A, has been shown to be a risk factor for the presentation of venous thrombosis.

We present the case of a 23-year-old non-smoking woman, a heterozygous carrier of a prothrombin gene mutation G20210A (hypercoagulability), who after confinement due to COVID-19 (impaired blood flow due to immobility) and use of hormonal patch contraceptives (endothelial abnormality), triggered deep vein thrombosis (DVT) / pulmonary thromboembolism (PE) that required hospital admission; and who, after rapid withdrawal of anticoagulant treatment, presented a second and a third episode of DVT. We highlight the usefulness of evaluating risk factors in G20210A heterozygous patients and the proper management of anticoagulation to avoid recurrences in patients susceptible to DVT / PE.

\section{Introduction}

Venous thromboembolic disease (VTD) is a common and life-threatening condition. The incidence of pulmonary thromboembolism (PE) is 1: 1000 inhabitants / year. It is more common in men and increases the risk 2 times per decade in those over 40 years of age. It causes $1 \%$ of hospital admissions and is considered the leading cause of in-hospital death [1]. In the prevention of VTD, it is essential to prevent deep vein thrombosis (DVT), since it will trigger PE, since up to $60-90 \%$ of the risk factors are identifiable, while only $20-30 \%$ of causes are attributed to genetic factors [1].

The impact of VTD on society is high as VTD is the third leading cause of cardiovascular mortality after ischemic heart disease and strokes. The incidence is 1: 1000 inhabitants / year; It is estimated that in the United States of America there are 94 fatal PE per 100,000 inhabitants / year and that 50,000 people die a year on average. Furthermore, $75 \%$ of people with DVT will develop a post-phlebitic or post-thrombotic syndrome [2].

Virchow's triad, still in force after being described in 1859, establishes that the following predisposing factors come into play in the pathogenesis of VTD: a) the state of hypercoagulability or thrombophilia; $b$ ) endothelial damage or injury (sometimes of pharmacological or mechanical origin); and c) venous stasis or impaired blood flow [1].

VTD is of multifactorial origin and genetic, environmental and acquired disease factors are described. Among genetic factors, the presence of hereditary thrombophilias stands out, such as the presentation of 
factor V Leiden (prevalence of 2 to $7 \%$ in the general population) and the presence of the mutation of the prothrombin gene G20210A (prevalence of 1 to $6 \%$ in the general population). Among the environmental factors, trauma, surgery or prolonged travel (with the consequent secondary immobility) have been described; acquired conditions (neoplasms, inflammatory bowel disease, paroxysmal nocturnal hemoglobinuria, autoimmune diseases) and the use of medications (thalidomide, L-asparaginase, hormonal therapy and contraceptives) are also related to TED. Pregnancy and the puerperium have also been proposed as risk factors for TED [3].

However, and despite current knowledge of the pathogenesis of thrombosis and Virchow's postulates, hereditary thrombotic disease is only diagnosed in $50 \%$ of cases; while in the other $50 \%$ of TED cases, no abnormality is ever diagnosed [5]. Therefore, it is accepted that venous stasis, generally caused by immobility syndrome, is the most important cause for developing TED, DVT, or PE [4].

The presentation of a new pandemic, such as COVID-19, which since its declaration on December 31, 2019 by the WHO, has spread so rapidly throughout the world to the point that it has caused many governments to have imposed very strict confinement measures with mobility restrictions. In Spain, since March 14, 2020, and until mid-June, most people have been confined to their homes with the absolute prohibition of doing any type of physical activity outdoors. This has led to a state of temporary immobility due to confinement [5].

The objective of this article is to present the case of a non-smoking young woman ( 23 years old), a carrier of the prothrombin gene mutation G20210A, who after immobility due to COVID-19 confinement and concomitant use of hormonal contraceptives, debuted with DVT / PE, and who, after prompt withdrawal of anticoagulant medication, relapses DVT in a second and a third episode; in addition to reviewing the bibliography and pharmacological management of DVT / PE, regarding the case.

\section{Clinical Case}

We present the clinical case of a 23-year-old non-smoking woman, with a medical history of asthma under aerosol treatment (Symbicort ${ }^{\circledR}$ ), use of the EVRA® contraceptive patch (Norelgestramine / Ethinylestradiol) and with a surgical history of rhinoseptoplasty, who attended the emergency room on time of confinement due to pandemic $(4 / 27 / 20)$ with pain in the left inguinal region, irradiated to the inner thigh and knee, of 3 days of evolution. The patient denies previous trauma, cough, dyspnea, or fever. On examination, pain on flexion and rotation of the left hip was observed, there was no evidence of erythema or induration at the venous level, but a small tumor of approximately $2-3 \mathrm{~cm}$ was palpated in the inguinal region. The ultrasound study revealed the complete occupation of echogenic material in the inguinal region and the absence of Doppler flow at the level of the common iliac vein and external iliac vein, extending to the superficial femoral vein (Figure 1a). The findings correspond to DVT in the common iliac, external iliac, and superficial femoral veins. Hospital admission was decided for treatment of DVT / PE and immediate removal of the contraceptive patch. Low molecular weight heparin was prescribed 
(LMWH Hepaxane ${ }^{\circledR} 80 \mathrm{mg}$ every $12 \mathrm{~h} \mathrm{SC}$, Acenocumarol $4 \mathrm{mg}$ (Sintrom ${ }^{\circledR}$ ) according to the guideline and strong compression stockings.

At 3 weeks after discharge, oral treatment (Acenocoumarol) was suspended, maintaining LMWH for a thrombophilia study, which revealed that the patient was a heterozygous carrier of a G20210A mutation of the prothrombin gene, which is why it was decided to continue anticoagulant treatment for 6 months, in order to consider indefinite, according to patient's evolution.

Seven months after the first episode of PE / DVT, the patient went to the hospital (11/17/20), reporting occasional pain in the left inguinal region, posterior region of the left thigh, and a sensation of dyspnea of several days of evolution. The patient maintained oral and subcutaneous anticoagulant treatment for 3 months, but then discontinued it. She kept compression stockings. On examination, tenderness was observed in the inguinal region and in the left posterior thigh, but a venous cord was not palpable in this region. In the analysis, the elevation of D-dimer levels $(544 \mathrm{ng} / \mathrm{mL}$ ) stood out. A new ultrasound study revealed echogenic material inside the superficial femoral vein, with the absence of Doppler flow, compatible with DVT at that level (Figure 1b). Once a second episode of DVT was diagnosed (clinical, analytical and ultrasound tests compatible), it was decided to restart anticoagulant treatment (LMWH Clexane ${ }^{\circledR}, 40$ mg / SC every 12h), keep compression stockings and follow-up by Internal Medicine and Hematology. It is recommended to avoid hormonal contraception, and in case of suspending anticoagulation, maintain prophylaxis if high-risk situations arise (surgery, immobilization or pregnancy). At 2 weeks of follow-up, D-dimer values decreased to $426 \mathrm{ng} / \mathrm{mL}$. It was decided to modify the anticoagulation regimen, prescribing Sintrom ${ }^{\circledR}$ according to the regimen, to maintain an INR between 2 and 3; Hibor ${ }^{\circledR}$ (5000U SC / every $24 \mathrm{~h}$ ), and continue with strong compression stockings.

Eleven months after the first episode (3/18/21), the patient returned to the hospital for pain in the left groin region, with characteristics similar to those presented in the 1st and 2nd episodes of DVT. She reported that the pain was worse at rest. On examination, no venous cord was observed, nor was induration palpable at that level. The INR revealed a level of 1.6 (lower than the recommended 2-3 level), and prothrombin activity of 50\% (normal range 70-120\%). A new ultrasound revealed findings compatible with DVT at the level of the superficial femoral vein (Figure 2). With the diagnosis of a 3rd episode of DVT, it was decided to suspend Sintrom ${ }^{\circledR}$ and prescribe LMWH Bemiparin at full doses (Hibor ${ }^{\circledR} 7500 \mathrm{IU} /$ $24 \mathrm{~h}$ ), and keep strong compression stockings.

One month after the 3rd episode, under review by Internal Medicine, it was decided to suspend Hibor ${ }^{\circledR}$, reintroduce Sintrom ${ }^{\circledR}$ maintaining an INR between 2-3, maintain strong compression stockings and avoid the use of hormonal contraceptives. As of today (October 2021), with this pattern, there have been no new episodes of DVT / PTSD.

\section{Discussion}

The review of the present clinical case has allowed us to observe that immobility due to COVID-19 confinement in a 23-year-old non-smoking female patient, heterozygous for the prothrombin gene 
G20210A, asymptomatic until the moment of immobility, and a user of hormonal contraception, are factors of risk significant enough to have triggered DVT / PE, up to three times, which highlights the multifactoriality of TED.

Thrombosis is a multifactorial entity that depends on genetic factors (such as the G20210A gene mutation in our case), and environmental factors (immobility and use of hormonal contraceptives) [6].

TED basically comprises two entities: a) DVT and b) PE; however, some authors consider c) thromboembolic cerebrovascular accident (CVA) as an entity of the same spectrum (TED). Many authors consider that PE is almost always secondary to DVT; therefore, they consider DVT / PE as a single entity [6]. The importance of these considerations is highlighted, since the incidence of PE is not negligible. The incidence of PE of 1: 100 older adults has been described; 1: 1000 young adults and 1: 100,000 children [6].

Rudolf Virchow described as early as 1859 the pathogenic triad for thromboembolic disease, highlighting three factors: a) venous stasis (immobility); b) endothelial injury; c) hypercoagulability or thrombophilia [7].

Regarding the first element of Virchow's triad (venous stasis and immobility), although the tourist class syndrome was described in 1958 as a temporary cause of immobility and a risk factor for developing DVT, it had already been described in 1940 PE by prolonged sitting in bomb shelters during the London bombings; and later in 1950, PE was described after prolonged sitting on long car trips, at work, or in the theater [7]. However, and despite the anecdotal of these publications, immobility is more frequent in bedridden patients, in casts, and even in paraplegics [7]. Recently, in 2003 e-thrombosis was described and in 2013 gamer-thrombosis due to prolonged sitting in front of a computer while playing video games. In 2006, the presentation of TED was also described in patients who, after immobility due to an earthquake in Japan, lived by car for 4-5 days. It is considered that 10 hours of sitting / day and 2 hours without getting up / day are risk factors for developing thrombosis. In fact, it is postulated that the new triad: a) obesity; b) sedentary lifestyle and; c) use of video games could be the cause of thrombophilia in young people between the ages of 13 and 18. Finally, in 2020, confinement due to COVID-19 has been described as a cause of temporary immobility that may be a new risk factor for developing DVT / PE [7].

This agrees with Franch-Llasat et al, who consider that thrombosis in the time of COVID-19 can be considered a collateral effect of confinement. In addition, they argue that the main risk factor for developing PE is immobility. In fact, it reports an incidence of 4 cases of PE in patients in a 10-day period of confinement, compared with the presentation of 7 cases of PE presented in a year without confinement (2019) [8]. They compared the cases with PE from 2020 and 2019, and found that the patients in confinement (2020) were younger, had fewer risk factors and only $15 \%$ had hereditary thromboembolic alterations (genetic factor). In fact, they suggest that up to $30 \%$ of PE are due to work or recreational immobility [8]. They concluded that, in young patients, immobility from confinement due to COVID-19 was the most important risk factor for developing TED [8]. This would explain the presentation of PE in the patient in our clinical case (immobility due to confinement). 
It is known that in $50 \%$ of thrombotic events (TED, DVT / PE) the causal factor cannot be identified. The other $50 \%$, transient or prolonged clinical or environmental factors have been detected that induce venous stasis, hypercoagulability and endothelial damage, as postulated by Virchow [8].

With regard to hypercoagulability or thrombophilia (second element of Virchow's triad), thrombophilia can be genetic or acquired. In the first place, Factor $V$ Leiden has been described as a genetic factor; the G20210A prothrombin gene mutation in second place; and the mutation of the enzyme methylene-tetrahydro-folate-reductase in third place [5, 6]. Anti-phospholipid syndrome, systemic lupus erythematosus, resistance to protein $\mathrm{C}$, hyperhomocystinemia, sticky platelet syndrome, and cancer have been described as acquired factors [6].

The mutation of the prothrombin gene G20210A is associated with an increased risk of venous thrombosis. Poor et al, in 1996, was the first to describe that the transposition of Guanine by Adenosine at position 20210 caused an increase in plasma prothrombin levels in carriers of this mutation. In heterozygotes (G / A), the prothrombin level increased by $30 \%$; while in homozygous ( $A$ / $A$ ), the levels increased by $70 \%$ [9]. The inheritance of the G20210A mutation is autosomal dominant. Its prevalence is $1-3 \%$ in the general population, $6 \%$ in patients with DVT, and $10 \%$ in families with a history of thrombophilia [10]. By race, the prevalence of the G20210A mutation in Caucasian Americans is 2\%; while in African Americans, Asians, Africans and Native Americans it is $0.5 \%$ [10]. This mutation increases the risk of developing TED by between 2 and 5 times, with the same behavior both by age and by sex [10].

With regard to endothelial injury / damage (third element of Virchow's triad), it has been reported that some drugs, including contraceptives, could inflame the intimal layer [4]. Cabrera-Payne et al have observed that the conjunction of the G20210A gene mutation and the presence of use of hormonal contraception and / or pregnancy are sufficient risk factors for the presentation of DVT / PE [10]. Del Valle-Rubido et al have described that the G20210A gene mutation associated with pregnancy / puerperium are sufficient risk factors for the presentation of postpartum ovarian venous thrombosis [10]. This reinforces the point that hormonal changes (pregnancy / puerperium) constitute a risk factor for the presentation of TED. This is also in agreement with Cabrera-Payne et al, who argue that in women heterozygous for the G20210A gene mutation, exposure to hormonal contraceptives significantly increases the risk of developing DVT and cerebral thrombosis [10]. Therefore, the thrombogenic effect of the mutated G20210A gene plus the appearance of modifiable risks such as the use of hormonal contraceptives, pregnancy or the puerperium, in addition to immobility (surgical or traumatic), could favor the presentation of thrombosis, as has occurred in the present clinical case [10].

Finally, the management of TED requires pharmacological and non-pharmacological measures. The use of strong compression stockings decreases post-phlebitic syndrome by $50 \%$, which appears in up to $60 \%$ of cases with thrombosis [1]. The mortality of PE is $30 \%$, therefore, the use of effective pharmacological measures is required. Anticoagulation begins with low molecular weight heparin (LMWH) and then to add Acenocoumarol (Sintrom) until maintaining an INR (International Normalized Ratio) between 2 and 3. After an idiopathic episode and if treatment was maintained between 3 and 6 months, the recurrence rate 
per year is $5-10 \%$. If there is a second episode of TED, the recurrence rate per year if anticoagulated for 6 months is $21 \%$, while it will be $2.6 \%$ if anticoagulated indefinitely [1]. Specialists suggest 6 months of anticoagulation after an idiopathic episode, one year after a second episode and anticoagulation for life if three episodes occur [1]. This would explain in our clinical case the observed recurrence of up to three episodes in one year, which suggests that the patient probably has to be anticoagulated indefinitely, given the existing hypercoagulable state (G20210A gene mutation).

Cabrera-Payne et al have shown that the use of LMWH in addition to oral antiagregants (aspirin) is effective in the management of TED in pregnant patients with a G20210A gene mutation [10].

\section{Conclusion}

Genetic predisposition (G20210A gene mutation) plus the presence of environmental factors (immobility due to COVID-19 confinement and simultaneous use of hormonal contraception) are factors that trigger TED. Of the Virchow triad, venous stasis (due to immobility) is the most important factor in developing thrombosis, especially in times of a COVID-10 pandemic. It is recommended that in young female patients, carriers of the G20210A prothrombin gene mutation, the risk of using hormonal contraceptives be carefully assessed to avoid the development of TED, DVT and / or PE.

\section{Declarations}

Funding: Authors declare no financial funding/support for the elaboration of this Case Report.

Conflict of interest/competing interest: The authors declare that they have no conflict of interest.

Consent to participate: Not applicable.

Consent for publication: For the publication of this Case Report, informed consent was obtained from the patient/legal representative included in the manuscript.

Ethical approval: the treatment provided to the patient was in accordance to the ethical guidelines of Declaration of Helsinsky (1975).

Availability of data and material: Not applicable.

Code availability: Not applicable.

Author's contribution: Conceptualization; drafting; investigation, supervision, writing, review, editing and translation: LCB and MEFC. Patients' treatment: LCB, ARG, GD, JAD, NDM, LGT, ELM. All authors have read and agreed to the published version of the manuscript.

\section{References}


1. Gómez, E. S., \& Cabañas, M. G. (2005). La enfermedad tromboembólica y su prevención. Enfermería en cardiología: revista científica e informativa de la Asociación Española de Enfermería en Cardiología, (34), 29-33.

2. Boccalon, H., Elias, A., Chalé, J. J., Cadene, A., \& Gabriel, S. (2000). Clinical outcome and cost of hospital vs home treatment of proximal deep vein thrombosis with a low-molecular-weight heparin: the Vascular Midi-Pyrenees study. Archives of internal medicine, 160(12), 1769-1773.

3. Hennessey-Calderón, D. C., Remolina-Murillo, N., \& Queremel-Milani, D. A. (2021). Trombofilia por hiperhomocisteinemia secundaria a mutación del gen MTHFR. Revista de Hematología, 22(1), 5661.

4. Sánchez, L. S., Cruz, G. J., Sáenz, P. C., Alfaro, V. R., \& Holst, H. F. (2007). Mutación 20210G/A del gen de la protrombina en una familia costarricense. Revista Médica de la Universidad de Costa Rica, 1(1), 28-34.

5. Vannini, L., Gómez, J. M. L., Quijada-Fumero, A., Pérez, A. B. F., \& Afonso, J. S. H. (2020). Embolia pulmonar aguda durante la cuarentena por COVID-19. Revista Española de Cardiología, 73(8), 680.

6. Hernández-Cuervo, H., Usme, S., \& Yunis, J. J. (2014). Genotipos frecuentemente asociados a trombofilias. Biomédica, 34(1), 132-142.

7. Lozano Sánchez, F. S. (2020). Trombosis venosa por falta" temporal" de movilidad. La COVID-19. Angiología, 72(3), 115-117.

8. Franch-Llasat, D., Mayor-Vázquez, E., Pedregosa-Díaz, J., Herrero-Redondo, M., Ortin-Font, X., \& RocheCampo, F. (2021). e-Thrombosis en época COVID-19. Efectos colaterales del confinamiento. Medicina Intensiva, 45(2), 122.

9. Bafunno, V., \& Margaglione, M. (2010). Genetic basis of thrombosis. Clinical Chemistry and Laboratory Medicine, 48, S41-51.

10. Cabrera-Payne, Y., Castillo-González, D., Rodríguez-Pérez, L., Tejeda-González, M., Fonseca-Polanco, C., \& Rubio Blanco, Y. (2015). Mutación g20210a del gen de la protrombina: presentación clínica en dos gestantes. Revista Cubana de Hematología, Inmunología y Hemoterapia, 31(4), 0-0.

\section{Figures}


A

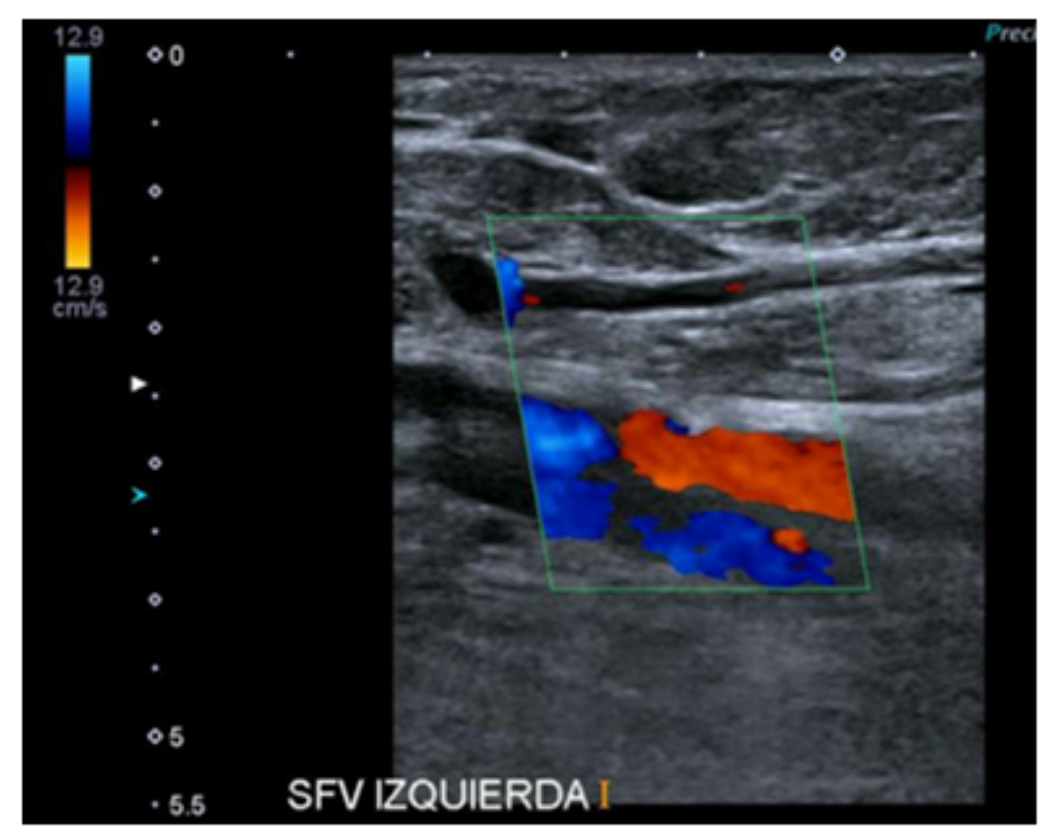

B

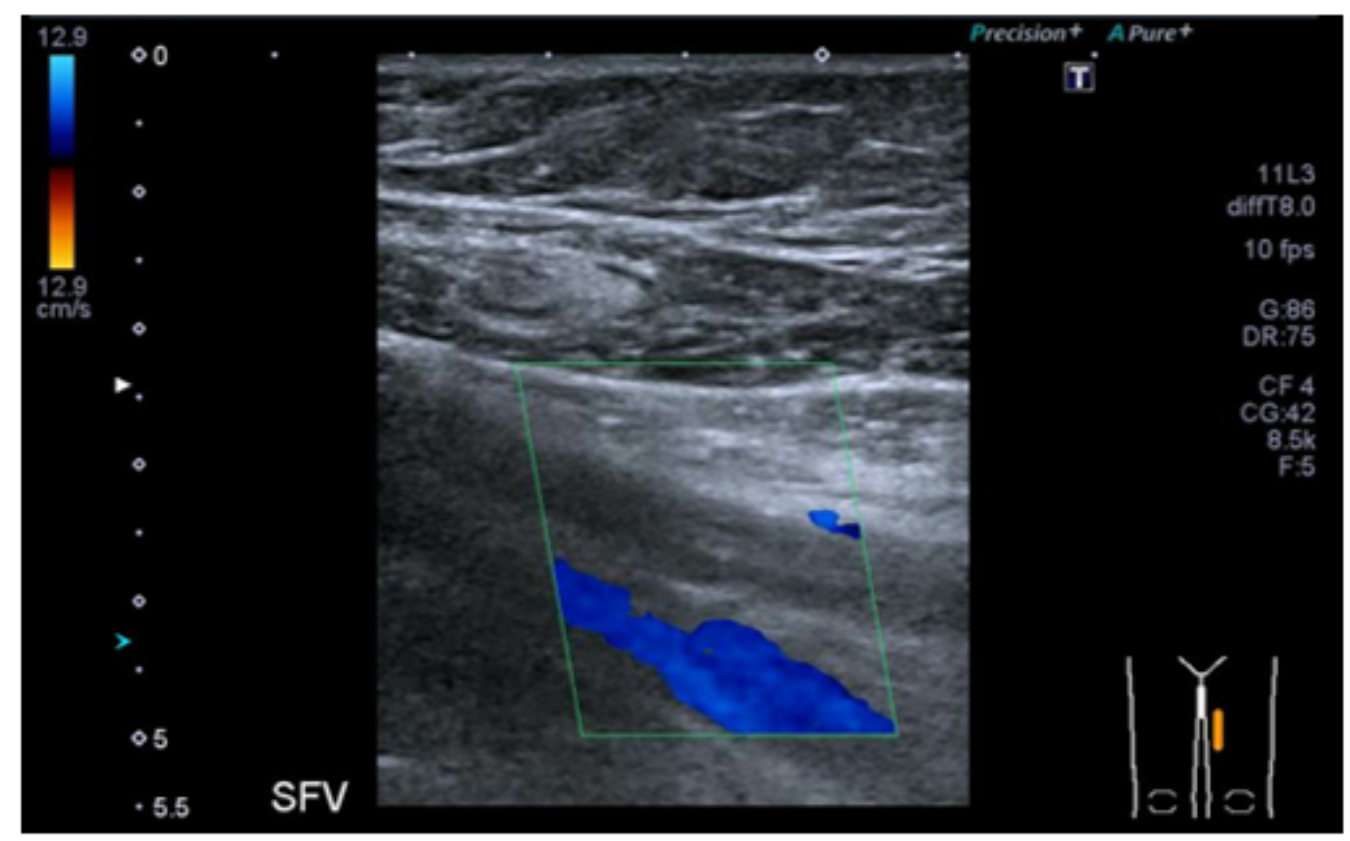

Figure 1

a. Color Doppler ultrasound demonstrates venous obstruction at the level of the left iliac vein and the superficial saphenous vein (first episode). b. Color Doppler ultrasound demonstrates venous obstruction at the level of the left superficial saphenous vein (second episode). 


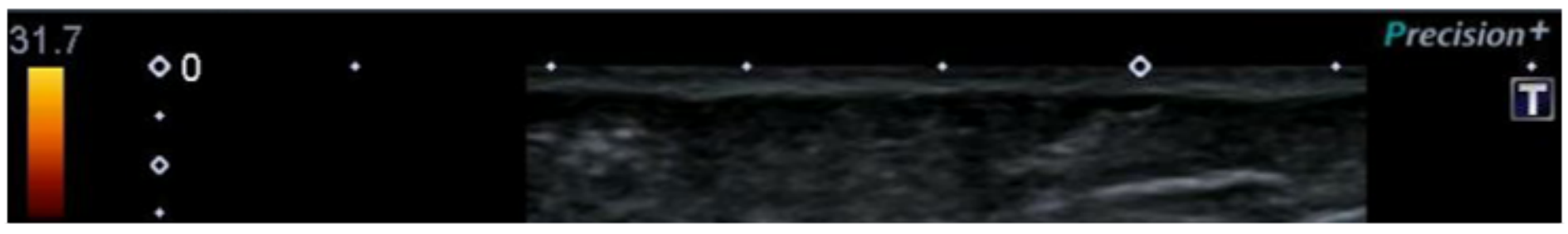

Figure 2

Color Doppler ultrasound demonstrates venous obstruction at the level of the left superficial saphenous vein (third episode) 